\title{
A limitation of the Cognitive Reflection Test: Familiarity
}

\author{
Stefan Stieger ${ }^{\text {Corresp., }} 1,2$, Ulf-Dietrich Reips ${ }^{1}$ \\ 1 Department of Psychology, University of Konstanz, Konstanz, Germany \\ 2 School of Psychology, University of Vienna, Vienna, Austria \\ Corresponding Author: Stefan Stieger \\ Email address: stefan.stieger@uni-konstanz.de
}

The Cognitive Reflection Test (CRT; Frederick, 2005) is a frequently used measure of cognitive vs. intuitive reflection. It is also a frequently found entertaining 'test' on the Internet. In a large age-stratified community-based sample $(N=2,272)$ we analyzed the impact of having already performed the CRT or any similar task in the past. Indeed we found that $44 \%$ of participants had experiences with these tasks, which was reflected in higher CRT scores (Cohen's $d=0.41$ ). Furthermore, experienced participants were different from naïve participants regarding their socio-demographics (younger, higher educated, fewer siblings, more likely single or in a relationship than married, having no children). The best predictors of a high CRT score were the highest educational qualification (4.62\% explained variance) followed by the experience with the task $(3.06 \%)$. Therefore, we suggest to use more recent multi-item CRTs with newer items and more elaborated test construction. 
1 Running head: COGNITIVE REFLECTION TEST

2 Word count: 5,045

3

4

5

6

A limitation of the Cognitive Reflection Test: Familiarity

8

Stefan Stieger ${ }^{1,2^{*}} \&$ Ulf-Dietrich Reips ${ }^{1}$

10

$11{ }^{1}$ Research Methods, Assessment, and iScience group, Department of Psychology, University of Konstanz, Germany

2 Department of Basic Psychological Research and Research Methods, School of Psychology, University of Vienna, Austria

15

16

17

18

19

20

21 * Corresponding author. Stefan Stieger, Department of Psychology, University of Konstanz,

22 Universitätsstraße 10, D-78464 Konstanz, Germany. E-mail: stefan.stieger@uni-konstanz.de 


\section{Abstract}

24 The Cognitive Reflection Test (CRT; Frederick, 2005) is a frequently used measure of cognitive 25 vs. intuitive reflection. It is also a frequently found entertaining 'test' on the Internet. In a large 26 age-stratified community-based sample $(N=2,272)$ we analyzed the impact of having already

27 performed the CRT or any similar task in the past. Indeed we found that $44 \%$ of participants had 28 experiences with these tasks, which was reflected in higher CRT scores (Cohen's $d=0.41$ ).

29 Furthermore, experienced participants were different from naïve participants regarding their

30 socio-demographics (younger, higher educated, fewer siblings, more likely single or in a

31 relationship than married, having no children). The best predictors of a high CRT score were the

32 highest educational qualification ( $4.62 \%$ explained variance) followed by the experience with the

33 task (3.06\%). Therefore, we suggest to use more recent multi-item CRTs with newer items and

34 more elaborated test construction. 


\section{Introduction}

The Cognitive Reflection Test (CRT) was introduced by Frederick (2005) and is

supposed to be a measure of cognitive reflection in contrast to intuition. It consists of three

mathematical/numerical text-based problems, which elicit first an intuitive (wrong) answer and

can only be solved when consciously thinking of the (not obviously) true answer. The theory

behind this task assumes that there are two distinct cognitive processes: a fast intuitive one and a

slow and rather reflective one (Epstein, 1994). Some researchers called them System 1 (i.e.,

spontaneous, instantly, effortlessly) and System 2 processes (i.e., effortful, motivated, reflected;

Stanovich \& West, 2000). To solve the CRT items, one has to ignore the first intention of the

System 1 processes and switch to System 2 processes to think intentionally about the correct

answer. The CRT has been frequently used and within many research topics (e.g., ideology:

Deppe, Gonzales, Neiman, Jacobs, Pahlke, Smith, \& Hibbings, 2015; superstitious and

paranormal beliefs: Pennycook, Cheyne, Seli, Koehler, \& Fugelsang, 2012).

The CRT has also been frequently studied from a methodological point of view. One

frequently researched topic is whether the CRT is rather a measure of numerical ability than of cognitive reflection. Using a mathematical modeling approach, Campitelli and Gerrans (2014)

found that the CRT is not only a measure of numerical ability, but also of rational thinking and open-minded thinking for males and of mathematical ability and rational thinking for women. Sinayev and Peters (2015) analyzed this issue in a large- $N$ multi-study design and found that numerical ability seems to be the key mechanism behind the CRT score (for a similar reasoning, see Welsh, Burns, \& Delfabbro, 2013). Furthermore, although the CRT could prove its usefulness in predicting normative decision making in (primarily) laboratory tasks (Toplak, 
60 personality and decision-making styles (Juanchich, Dewberry, Sirota, \& Narendran, 2016).

61 Some researchers suggested an alternative score of intuition (in contrast to reflection;

62 e.g., Brosnan, Hollinworth, Antoniadou, \& Lewton, 2014), but recent research found that the

63 reflective score still predicts behavior (e.g., intuitive-analytic cognitive styles) better than the

64 intuitive score (Pennycook, Cheyne, Koehler, \& Fugelsang, 2016). Because the CRT has only

65 three items, it often lacks high reliability values (range between .60 and .74; Weller, Dieckmann,

66 Tusler, Mertz, Burns, \& Peters, 2013; Liberali, Reyna, Furlan, Stein, \& Pardo, 2011; for a short

67 review, see Campitelli \& Gerrans, 2014). Therefore, multi-item revisions of the CRT with higher

68 reliability values have been introduced (e.g., 7 items: Toplak et al., 2014; 5 items: Gòmez-

69 Chacòn, Garcìa Madruga, Rodrìguez, Vila, \& Elosùa, 2012), some with psychometric properties

70 scrutinized by using Item Response Theory (6 items: Primi, Morsanyi, Chiesi, Donati, \&

71 Hamilton, 2015), which come with the additional advantage of being less susceptible to floor and

72 ceiling effects. To sum up, although some methodological topics are still under debate, the CRT

73 keeps developing and is used widely.

74 The CRT's frequent use and constant further development might explain its popularity,

75 the Web of Science lists 386 citations and Google Scholar lists 1,286 citations. All the more,

76 several well-known books cite this task (e.g., Kahneman, 2011) and the CRT is a frequently

77 found 'test' on the Internet where people can try to solve the items in a game-like competitive

78 manner with the aim of being better than their friends and/or peers. For example, the search term

79 'Cognitive Reflection Test' elicits 9,130 search results and the first few words of the first item of

80 the test ('a bat and a ball cost...') creates 8,570 hits on the Internet (German version 'ein

81 Schläger und ein Ball...'2,970 hits). Recent reports about people working on Amazon's

82 Mechanical Turk platform (i.e., a microjob web platform also called MTurk; Buhrmester, 
83 Kwang, \& Gosling, 2011) state that the CRT items are the ones which are probably most often

84 asked in MTurk projects. ${ }^{1}$

85 We found one study that investigated the influence of popularity on CRT results. Brañas-

86 Garza, Kujal, and Lenkei (2015) performed a meta-analysis about the CRT and also included a

87 cross-temporal meta-analysis, where they correlated the mean CRT score (per year), with the

88 respective year of publication. Overall, they found some support for their assumption that, with

89 time, CRT scores are increasing and this effect seems to be driven by online studies (where

90 participants have the possibility to look up the correct answer) although effect sizes were low.

91 However, in this cross-temporal meta-analysis the moderator variable of having already

92 performed the CRT was not explicitly assessed, rather it was assumed that in later samples

93 participants might have already performed such tasks and are more experienced (Brañas-Garza et

94 al., 2015).

95 The CRT obviously only works well if participants are not aware of the rationale behind

96 the task, i.e., that the CRT items elicit a spontaneous, intuitive answer and that this answer is not

97 the correct one. Therefore, we asked whether the obvious prominence of the task could have an

98 effect on the CRT's outcome (for a similar reasoning, see Toplak et al., 2014). If participants are

99 aware of the rationale, they can consciously overcome their first intuition and think about the

100 items more thoroughly knowing that the correct answer is probably not the first answer that came

101 to mind. If this assumption is correct, then participants who have already performed the CRT (or

102 any other similar task) in the past, should have higher CRT scores than participants doing the

103 CRT for the very first time.

104 To sum up, we investigated whether having already performed the CRT or any similar

${ }^{1}$ http://www.pbs.org/newshour/updates/inside-amazons-hidden-science-factory/ 
105 task (e.g., multi-item versions of the CRT: Toplak et al., 2014) in the past has an effect on the

106 CRT's outcome (mean difference, floor and ceiling effects) and whether differences in the

107 sample composition regarding socio-demographics (e.g., sex, age, highest educational

108 qualification, current relationship status) influence this effect.

\section{Method}

\section{Participants}

111 Participants $(\mathrm{N}=2,272)$ were recruited by word-of-mouth through friends, relatives, and

112 friends-of-friends of several research assistants following a convenience sampling approach.

113 Participants filled in the questionnaire wherever they were approached (e.g., at home, at the

114 university). The final sample was age-stratified and constitutes German-speaking volunteers

115 (predominantly Austria and Germany) from all walks of life, $M_{\text {age }}=39.8$ years, $S D=17.7$;

$11656.6 \%$ women. We used six different age-strata $(18-25,26-30,31-40,41-50,51-60,61+)$

117 with the aim of an equal number of participants in each strata in the final sample. In terms of

118 highest educational qualification, $4 \%$ had not completed primary education, $15 \%$ had completed

119 primary education, $23 \%$ had an apprenticeship diploma, 34\% had completed secondary

120 education, and 24\% had a university degree. Participants' current relationship status was: $24 \%$

121 single, $29 \%$ in a relationship, 38\% married, $4 \%$ divorced, $4 \%$ widowed, and 1\% stated a different

122 relationship status. Almost half of participants had children (46\%), 26\% were currently smoker,

123 and had on average 1.8 siblings (Median $=1$; range 0 to $11 ; 13 \%$ were the only child in their

124 family).

125 Materials

126 Cognitive Reflection Test (CRT). The CRT (Fredericks, 2005) is a measure of cognitive in

127 contrast to intuitive reflection. It contains three problems, which first elicit a spontaneous, but 
128 mathematically wrong answer. Only individuals who overcome this first intention and

129 deliberately think about the correct answer can solve the problems (e.g., first problem "A bat and

130 a ball cost $\$ 1.10$ in total. The bat costs $\$ 1.00$ more than the ball. How much does the ball cost?

131 cents."; intuitive answer $=10$ cents; correct answer $=5$ cents). The CRT has no time limit

132 and the total score is calculated as the number of correct answers (range 0 to 3 ; for descriptive

133 results, see Table 1). ${ }^{2}$ Directly after the three CRT items, we asked participants whether they had

134 done these tasks or any similar ones before (yes/no).

135

136 Table 1.

137 Success rate of the CRT items, results from the present study.

\begin{tabular}{llllllll}
\hline & \multicolumn{6}{c}{ Empirical results [\%] } \\
\cline { 2 - 8 } & Correct & Wrong & Correct & Wrong & Wrong (but & Missing & Sum \\
& answer & (intuitive) & & (intuitive & not & & \\
& & answer & & answer) & intuitive & & \\
& & & & & answer) & & \\
CRT Item 1 & 5 & 10 & $28.3 \%$ & $68.1 \%$ & $2.3 \%$ & $1.3 \%$ & $100.0 \%$ \\
CRT Item 2 & 5 & 100 & $55.0 \%$ & $33.1 \%$ & $10.0 \%$ & $1.9 \%$ & $100.0 \%$ \\
CRT Item 3 & 47 & 24 & $53.0 \%$ & $34.9 \%$ & $8.0 \%$ & $4.1 \%$ & $100.0 \%$
\end{tabular}

Note. CRT $=$ Cognitive Reflection Test.

139 Item 1: "A bat and a ball cost $€ 1.10$ in total. The bat costs $€ 1.00$ more than the ball. How much

140 does the ball cost? cents"

\footnotetext{
${ }^{2}$ We have also tried the intuitive score as an alternative (for a recent comparison, see Pennycook et al., 2015), but found very little differences in the results. This is probably due to the rather low rate of participants who gave a wrong non-intuitive answer (see Table 1).
} 
141 Item 2: "If it takes 5 machines 5 minutes to make 5 widgets, how long would it take 100

142 machines to make 100 widgets? ___ minutes"

143 Item 3: "In a lake, there is a patch of lily pads. Every day, the patch doubles in size. If it

144 takes 48 days for the patch to cover the entire lake, how long would it take for the patch to cover

145 half of the lake? days"

146 Demographics. Participants were asked about their sex, age, highest educational qualification,

147 and current relationship status. Because we were interested whether there are general differences

148 between those participants having already performed the CRT or any similar task in the past and

149 those without experience, we also asked further specific demographics without having any

150 specific research question in mind only to give a more complete picture of potential differences

151 in demographics, i.e., whether or not they have children (yes/no), smoke (yes/no), and how many

152 siblings they have, if any.

153 Procedure

154 Participants gave their informed consent, completed the CRT along with several other

155 measures that were not part of this study, and finally provided demographic details. For the

156 purpose of anonymity, each questionnaire was put into an envelope and thrown into a box. All

157 participants took part on a voluntary basis and were not financially remunerated for participation.

158 Dominance analysis

159 When calculating linear regressions, multicolinearity (i.e., intercorrelation between

160 predictors) is a problem when it turns out to be substantial. To quantify multicolinearity,

161 statistical packages calculate the so-called Variance Inflating Factors (VIFs). In the present

162 study, multicolinearity was foreseeable, because demographic variables usually correlate (e.g.,

163 participant age is correlated with the highest educational qualification and having children or 
164 not).

165 To account for multicolinearity, we decided to additionally conduct a dominance analysis

166 (Azen \& Budescu, 2003; Budescu, 1993). Dominance analyses have the advantage of assessing

167 the importance of each predictor relative to the other predictors in the regression model. This is

168 realized by looking at the contribution of each single predictor in the linear model not only in

169 conjunction with other predictors, but also in isolation. Practically, all possible combinations of

170 predictors are used to calculate partial, direct, and total effect parts by decomposing the total $R^{2}$

171 (explained variance). The partial effects come from all possible combinations of predictors on

172 the outcome measure by excluding either one or more predictors from the model. The direct

173 effect is the independent contribution without the other predictors in the model (i.e., zero-order

174 correlation with the outcome measure). The total effect represents the classical multiple linear

175 regression when all predictors are included in the model at once. The outcomes of the dominance

176 analysis are $R^{2}$ values for each predictor, which are adjusted for shared variances with other

177 predictors (i.e., representing the real explained variance). In the present study, dominance

178 analyses were calculated using the R package 'yhat' (Nimon \& Oswald, 2013; for a recent

179 application, see Stieger, Burger, Schiller, Schulze, \& Voracek, 2014).

180 Ethics

181 The present study was conducted in accordance with the principles of the Declaration of

182 Helsinki and with institutional guidelines of the School of Psychology, University of Vienna.

183 Furthermore, the present study followed the Guidelines for ethical conduct of behavioral projects

184 involving human participants proposed by the American Psychological Association. According

185 to the institutional guidelines of the University of Vienna, Austria

186 (http://satzung.univie.ac.at/ethikkommission-der-universitaet-wien/), approval by an ethics 
187 committee was not necessary because the study did not affect the physical or psychological

188 integrity, the right for privacy, or other personal rights or interests (see §2(1)). All participants

189 gave verbal informed consent after having received a written description of the study. Data

190 collection was anonymous and no harmful procedures were used.

\section{Results}

\section{Descriptives}

Out of the overall sample of 2,272 participants, 135 (5.9\%) did not answer at least one of

194 the three CRT questions (percentage of missing answers, see Table 1) or did not state whether or

195 not they had experience with the test $(n=23)$. Data from these participants were excluded from

196 further analyses, leading to a final sample size of $N=2,137$. In 45 cases (2.1\%), participants

197 misstated an obviously correct answer with the first item of the CRT by ignoring the fact that the

198 result should be stated in Cent not in Euros (e.g., participants stated 0.05 instead of 5). These

199 inconsistencies were solved and these cases included. We found no inconsistencies for the

200 second and third item of the CRT. Descriptive statistics of the CRT items can be found in Table 2011.

202 The question whether or not participants had already performed the CRT or any similar 203 task in the past were answered with "Yes" by almost half of participants $(44.9 \%, n=959)$. We 204 will differentiate between naïve and experienced participants in the following sections. The 205 reliability estimate of the CRT for the experienced participants was slightly higher than the one 206 for the naïve participants (Cronbach $\alpha=.657$ vs. .595, respectively).

207 Mean differences: Are there differences between naïve and experienced participants?

208 As can be seen from Table 2, there were several differences between the participant 209 groups, not only regarding the CRT but also regarding several demographic variables. First of 
210 all, as hypothesized experienced participants had significantly higher CRT scores than naïve

211 participants (low-to-medium effect size; Cohen, 1988). This experience effect affected all CRT

212 items more or less to the same extent (Odds Ratios ORs between 1.66 and 2.05).

213 
215 Table 2.

216 Differences between experienced and naïve participants regarding the variables under

217 investigation.

\begin{tabular}{|c|c|c|c|c|}
\hline & Experienced & Naïve & & \\
\hline & $M(S D)$ & $M(S D)$ & $t$-test & Cohen's $d$ \\
\hline CRT sum score & $1.65(1.11)$ & $1.21(1.06)$ & $9.38 * * *$ & 0.41 \\
\hline Age & $35.0(16.00)$ & $43.4(17.93)$ & $11.36^{* * *}$ & 0.50 \\
\hline Education & $3.8(1.12)$ & $3.5(1.10)$ & $7.11 * * *$ & 0.31 \\
\hline \multirow[t]{2}{*}{ Number of siblings } & $1.7(1.46)$ & $1.9(1.61)$ & $3.44 * *$ & 0.15 \\
\hline & \multicolumn{2}{|c|}{ Standardized residuals } & $\chi^{2}$ & $O R[C I]$ \\
\hline CRT Item 1 & $-3.0 / 4.7$ & $2.7 /-4.2$ & $56.25 * * *$ & $2.05[1.70,2.48]$ \\
\hline CRT Item 2 & $-3.5 / 2.8$ & $2.9 /-2.5$ & $32.86 * * *$ & $1.66[1.40,1.98]$ \\
\hline CRT Item 3 & $-4.3 / 3.9$ & $3.9 /-3.5$ & $61.56 * * *$ & $2.01[1.69,2.39]$ \\
\hline $\operatorname{Sex}[\mathrm{m} / \mathrm{f}]$ & $0.8 /-0.7$ & $-0.7 / 0.6$ & 1.97 & $0.88[0.74,1.05]$ \\
\hline \multirow[t]{2}{*}{ Relationship status ${ }^{1)}$} & $2.7 / 3.8 /-4.8 /$ & $-2.4 /-3.4 / 4.3 /$ & $88.87 * * *$ & $C C=0.2$ \\
\hline & $-0.4 /-2.0 / 0.5$ & $0.4 / 1.8 /-0.4$ & & \\
\hline Own children [yes/no] & $-5.2 / 4.9$ & $4.7 /-4.4$ & $92.74 * * *$ & $2.36[1.98,2.82]$ \\
\hline Smoker [yes/no] & $-0.7 / 0.4$ & $0.7 /-0.4$ & 1.35 & $1.12[0.92,1.36]$ \\
\hline \multicolumn{5}{|c|}{ Note. ${ }^{1)}$ Coding of relationship status: single, in a relationship, married, divorced, widowed, } \\
\hline
\end{tabular}


Furthermore, experienced and naïve participants differed in their demographics.

224 Experienced participants were younger, had a higher education, fewer siblings, no children, and

225 were more likely single or in a relationship than married (see standardized residuals in Table 2).

226 The difference between experienced and naïve participants regarding the CRT score

227 becomes even more pronounced for different educational levels (see Table 3). Compared to the

228 university samples presented by Frederick (2005), participants in the present study having 229 experience with the CRT or any similar tasks and having an apprenticeship diploma as highest 230 educational level, performed slightly better than Harvard students (1.50 vs. 1.43). The same 231 applies to participants with secondary education, they were similar to Princeton students (1.65 232 vs. 1.63), and participants with a university degree performed almost as well as Massachusetts 233 Institute of Technology (MIT) students (1.99 vs. 2.18). Furthermore, floor and ceiling effects 234 became relevant. About one third of experienced participants with secondary education or a 235 university degree reached the highest score possible $(=3)$, whereas again one third of 236 experienced participants with lower education did not solve any CRT item. 
239 Table 3.

240 Differences in CRT scores between experienced and naïve participants separated by highest

241 educational qualification.

\begin{tabular}{|c|c|c|c|c|c|c|}
\hline & \multirow{2}{*}{$\begin{array}{l}\text { Mean } \\
\text { CRT score } \\
(S D)\end{array}$} & \multicolumn{4}{|c|}{ CRT score $[\%]$} & \multirow[t]{2}{*}{$N$} \\
\hline & & 0 & 1 & 2 & 3 & \\
\hline \multirow[t]{2}{*}{ Not completed } & $1.16(1.08)$ & $36 \%$ & $25 \%$ & $25 \%$ & $14 \%$ & 44 \\
\hline & $1.22(1.03)$ & $30 \%$ & $32 \%$ & $24 \%$ & $14 \%$ & 37 \\
\hline \multirow[t]{2}{*}{ Primary education } & $0.93(0.97)$ & $43 \%$ & $29 \%$ & $20 \%$ & $8 \%$ & 191 \\
\hline & $1.16(1.17)$ & $43 \%$ & $16 \%$ & $23 \%$ & $18 \%$ & 122 \\
\hline \multirow[t]{2}{*}{ Apprenticeship diploma } & $1.06(1.03)$ & $39 \%$ & $27 \%$ & $23 \%$ & $11 \%$ & 359 \\
\hline & $1.50(1.12)$ & $26 \%$ & $21 \%$ & $30 \%$ & $23 \%$ & 121 \\
\hline \multirow[t]{2}{*}{ Secondary education } & $1.31(1.05)$ & $29 \%$ & $27 \%$ & $28 \%$ & $16 \%$ & 344 \\
\hline & $1.65(1.11)$ & $22 \%$ & $20 \%$ & $29 \%$ & $29 \%$ & 390 \\
\hline \multirow[t]{2}{*}{ University degree } & $1.55(1.07)$ & $23 \%$ & $23 \%$ & $32 \%$ & $22 \%$ & 236 \\
\hline & $1.99(0.64)$ & $10 \%$ & $17 \%$ & $37 \%$ & $36 \%$ & 285 \\
\hline
\end{tabular}

242 Note. First-line entry = naïve participants, second-line entry = experienced participants.

244 In a next step, we calculated a linear regression with the CRT score as the dependent

245 measure to better quantify the influence of the CRT knowledge and participants' demographics

246 onto the CRT score. Because some of the predictors are intercorrelated (e.g., age with current

247 relationship status, highest educational qualification, and having children), multicolinearity can

248 be assumed. This is a problem for linear regressions, because the influence of predictors onto the 
249 dependent measure are not pure, they may depend on other predictors. Therefore, we also

250 calculated a dominance analysis. Results can be found in Table 4. Variance Inflation Factors

251 (VIFs), which indicate multicolinearity, were between 1.034 and 17.692 (following current

252 practices, VIFs higher than 10 are regarded as problematic; O'Brien, 2007).

253

254 Table 4.

255 Results of the linear regression and dominance analysis with the CRT score as the dependent 256 measure.

\begin{tabular}{|c|c|c|c|}
\hline & $\beta$ & $\begin{array}{l}\text { Zero-order } \\
\text { correlation } r\end{array}$ & $\begin{array}{l}\text { Dominance } \\
R^{2}[\%]\end{array}$ \\
\hline Age & -.018 & -.028 & $0.05 \%$ \\
\hline Education & $.203 * * *$ & $.239 * * *$ & $4.62 \%$ \\
\hline Number of siblings & -.001 & $-.048 *$ & $0.08 \%$ \\
\hline Sex & $-.138 * * *$ & $-.141 * * *$ & $1.88 \%$ \\
\hline Own children & .011 & -.026 & $0.03 \%$ \\
\hline Smoker & $-.091 * * *$ & $-.088^{* * * *}$ & $0.80 \%$ \\
\hline CRT experience & $.158^{* * *}$ & $.198^{* * *}$ & $3.06 \%$ \\
\hline \multicolumn{4}{|l|}{ Relationship status } \\
\hline Single & -.016 & $-.055^{* *}$ & $0.19 \%$ \\
\hline In a relationship & .077 & $.084 * * *$ & $0.32 \%$ \\
\hline Married & .062 & .005 & $0.07 \%$ \\
\hline Divorced & .015 & -.016 & $0.02 \%$ \\
\hline Widowed & -.003 & $-.069 * *$ & $0.20 \%$ \\
\hline
\end{tabular}


257 Note. $F(12,2070)=22.03, p<.001 ;$ adj. $R^{2}=10.8 \%$

258 Coding of Sex: 1..male, 2..female; Coding of 'own children' and smoker: 0..no, 1...yes

$* p<.05, * * p<.01, * * * p<.001$ (two-tailed).

260 The impact of multicolinearity on the beta weights can also be tested by calculating

261 Spearman rank-order correlations between dominance weights (which should be true values

262 adjusted for intercorrelations) and the absolute values of beta weights. In case of no

263 multicolinearity, order ranks of dominance weights should resemble the order ranks of beta

264 weights (i.e., perfect rank-order correlation of 1). If the rank-order correlation deviates from 1,

265 the more likely multicolinearity is present. In fact, the rank-order correlation was below $1\left(r_{\mathrm{sp}}=\right.$

$266.76, p<.01)$. Therefore, the dominance weights should be given preference over beta weights.

267 As can be seen in Table 4, the best predictors of the CRT score were the highest

268 educational qualification, followed by CRT experience, participant's sex (male higher scores),

269 and being a smoker (smokers had lower CRT scores, i.e. scored rather intuitive than reflective).

270 Most of the overall explained variance could be attributed to the first two predictors (highest

271 education qualification, CRT experience: $7.68 \%$ in total). We repeated the analyses for each

272 CRT item, but the pattern of results remained constant. The only exception was that for the CRT

273 Item 1, age was also significant (the lower the age, the higher the score; $\beta=-.095$; detailed

274 results omitted for brevity).

275

276

277

278

279

\section{Discussion}

In the present study we could clearly show that having prior experience with the CRT or any similar task has a substantial influence on the CRT score $(d=0.41)$. CRT experience was one of the best predictors of the CRT score (3.06\% explained variance), along with the highest educational qualification (4.62\%). 
they were also different from naïve participants regarding their socio-demographics (e.g., young,

282 lower educated, being rather single or in a relationship than married, and having no children).

283 Furthermore, floor and ceiling effects were prevalent. Almost one third of higher educated

284 (secondary education, university) experienced participants reached the highest possible score,

285 whereas one third of the lower educated participants did not solve any CRT item at all.

286 This suggests that the CRT in its original 3-item form (Frederick, 2005) is not only

287 limited by familiarity, it is also limited by range restrictions. Although the items seem to be of

288 medium difficulty, the classical CRT is not suitable for the highly educated (because they solve

289 all items) as well as the lowly educated (because they solve none of them).

290 Therefore, we strongly recommend using recent multi-item CRTs (including new

291 intuitive vs. reflection items; Primi et al., 2015; Toplak et al., 2014). This recommendation has

292 several implications. First, following our recommendation should lower the probability that

293 participants have experience with the CRT's items, at least for some time. When contrasting

294 results based on old and new items, one can evaluate a possible influence from CRT experience

295 (at least with the original CRT). If one wants to avoid the original CRT items entirely, Toplak et

296 al. (2014) also introduced a 4-item version of the CRT including only new items. Second, multi-

297 item CRTs have higher measurement reliability. Currently the CRT's reliability is rather low - in

298 the present sample, naïve participants produced a rather low reliability of Cronbach $\alpha=.595$.

299 Also, it could be that reliability estimates from past studies are impaired by CRT experience as

300 well, i.e., the real reliability estimate is actually even lower. Using reliable multi-item CRTs

301 should also raise the possibility to find substantial and meaningful effects in future research 
302 projects because increased measurement reliability leads to larger effect sizes (for a discussion,

303 see LeBel \& Paunonen, 2011) as well as less range restrictions.

304 The present results are also of interest regarding another effect - the Flynn effect (i.e.,

305 secular IQ gains; for an overview see Williams, 2013). The Flynn effect is the substantial

306 increase in intelligence (fluid and crystallized) test scores over time (from $\sim 1930$ until now). The

307 reasons for this effect are still under debate, but it seems to be a mixture of several influences

308 such as real IQ gains through education or increases in test-specific skills (e.g., Jensen, 1998).

309 For the CRT, the same effect of increasing test scores has been found by Brañas-Garza et al.

310 (2015) in their cross-temporal meta-analysis. Initially, this could mean that people indeed are

311 becoming more rational and reflective (compared to intuitive) over time. But the authors also

312 found that this effect was driven by online samples, i.e., the effect might be driven by

313 participants looking up the correct answers on the Internet. The present study extends and

314 clarifies this supposition, that indeed the prevalence rate of knowing the CRT (and similar tasks)

315 is high and indeed prior experience with the task substantially raises the CRT score. Hence, the

316 gain in CRT scores is due to gains in test-specific skills rather than a gain in rational thinking.

\section{Limitations}

318 In the present study only German-speaking volunteers were recruited, therefore it is

319 unclear how the found results apply to other communities. We believe that for English-speaking

320 countries the CRT experience effect might even be more prevalent. First of all, the CRT was

321 originally published in English, so there was more time for items to become well-known in

322 English. Second, as far as we know, the CRT items (especially the first bat-and-ball item) are

323 frequently used in introductory courses and classroom presentations in the US and UK. In the

324 future, it would be interesting to analyze potential experience effects with the CRT items in other 
325 languages (e.g., Spanish; Gòmez-Chacòn et al., 2012).

326 Furthermore, in the present study we only asked about whether or not participants have

327 any experience with the CRT items or similar tasks, but we did not ask how much experience

328 they have and when they made this experience (long time ago vs. very recently). Assessing these

329 two additional variables would make it possible do draw a more nuanced picture about the

330 influence of CRT experience onto CRT scores.

331 Conclusion

332 To sum up, we think that a methodologically well-developed CRT is vital in order to

333 settle debates about the CRT (e.g., its dependency on numerical ability and correlation with

334 intelligence). Currently the classical CRT (Frederick, 2005) is limited by familiarity because it is

335 frequently found on the Internet and is frequently used in introductory courses and classroom

336 presentations. Furthermore, it is limited by range restrictions due to its 3 -item form. With the

337 present study we could show that experience strongly affects the CRT and a revalidation of the

338 task is therefore indicated. Fortunately, there are already encouraging new developments

339 regarding the CRT (e.g., Primi et al., 2015; Toplak et al., 2014) which should be given

340 preference over the classical CRT. 


\section{References}

342 Azen, R., \& Budescu, D. V. (2003). The dominance analysis approach for comparing predictors 343 in multiple regression. Psychological Methods, 8, 129-148.

344 Brañas-Garza, P. Kujal, P., \& Lenkei, B. (2015). Cognitive Reflection Test: Whom, how, when.

345 Munich Personal RePEc Archive paper no. 68049. https://mpra.ub.uni-

$346 \quad$ muenchen.de/68049/

347 Brosnan, M., Hollinworth, M., Antoniadou, K., \& Lewton, M. (2014). Is empathizing intuitive doi:10.1016/j.paid.2014.03.006

Budescu, D. V. (1993). Dominance analysis: A new approach to the problem of relative importance of predictors in multiple regression. Psychological Bulletin, 114, 542-551.

352 and systemizing deliberative? Personality and Individual Differences, 66, 39-43.

Buhrmester, M., Kwang, T., \& Gosling, S. D. (2011). Amazon's Mecahnical Turk: A new source of inexpensive, yet high-quality, data? Perspectives on Psychological Science, 6, 3-5. doi:10.1177/1745691610393980

Campitelli, G., \& Gerrans, P. (2014). Does the Cognitive Reflection Test measure cognitive reflection? A mathematical modelling approach. Memory and Cognition, 42, 434-447. doi: $10.3758 / \mathrm{s} 13421-013-0367-9$

Cohen, J. (1988). Statistical power analysis for the behavioral sciences (2nd ed.), New Jersey: Lawrence Erlbaum.

Deppe, K. D., Gonzales, F. J., Neiman, J. L., Jacobs, C., Pahlke, J., Smith, K. B., \& Hibbings, J. R. (2015). Reflective liberals and intuitive conservatives: A look at the Cognitive Reflection Test and ideology. Judgment and Decision Making, 10, 314-334

Epstein, S. (1994). Integration of the cognitive and psychodynamic unconscious. American 
Psychologist, 49, 709-724.

365 Frederick, S. (2005). Cognitive reflection and decision making. Journal of Economic

366 Perspectives, 19, 25-42.

367 Gòmez-Chacòn, I., Garcìa Madruga, J. A., Rodrìguez, R., Vila, J. O., \& Elosùa, R. (2012).

368 Mathematical beliefs and cognitive reflection: Do they predict academic achievement? In

369 B. Roesken \& M. Casper (Eds.), Proceedings of the MAVI-17 Conference, September 17-

370 20, 2011, Current state of research on mathematical beliefs (pp. 64-73). Ruhr-Universität

$371 \quad$ Bochum, Germany.

372 Jensen, A. R. (1998). The g factor: The science of mental ability. Westport, CT: Praeger.

373 Juanchich, M., Dewberry, C., Sirota, M., \& Narendran, S. (2016). Cognitive reflection predicts

374 real-life decision outcomes, but not over and above personality and decision-making styles.

375 Journal of Behavioral Decision Making. doi:10.1002/bdm.1875

376 Kahneman, D. (2011). Thinking, fast and slow. New York: Farrar, Straus and Giroux.

377 LeBel, E. P., \& Paunonen, S. V. (2011). Sexy but often unreliable: The impact of unreliability on

378 the replicability of experimental findings with implicit measures. Personality and Social

$379 \quad$ Psychology Bulletin, 37, 570-583. doi:10.1177/0146167211400619

380 Liberali, J. M., Reyna, V. F., Furlan, S., Stein, L. M., \& Pardo, S. T. (2011). Individual

381 differences in numeracy and cognitive reflection, with implications for biases and fallacies

382 in probability judgment. Journal of Behavioral Decision Making, 25, 361-381.

383 Nimon, K., \& Oswald, F. L. (2013). Understanding the results of multiple linear regression:

384 Beyond standardized regression coefficients. Organizational Research Methods, 16, 650-

385 674. doi:10.1177/1094428113493929

386 O’Brien, R. M. (2007). A caution regarding rules of thumb for variance inflation factors. Quality 
and Quantity, 41, 673-690. doi:10.1007/s11135-006-9018-6

388

389

390

391

392

393

394

395

396

397

398

399

400

401

402

403

404

405

406

407

408

409

Pennycook, G., Cheyne, J. A., Koehler, D. J., \& Fugelsang, J. A. (2016). Is the Cognitive Reflection Test a measure of both reflection and intuition? Behavior Research Methods. doi:10.3758/s13428-015-0576-1

Pennycook, G., Cheyne, J. A., Seli, P., Koehler, D. J., \& Fugelsang, J. A. (2012). Analytic cognitive style predicts religious and paranormal belief. Cognition, 123, 335-346. doi:10.1016/j.cognition.2012.03.003

Primi, C., Morsanyi, K., Chiesi, F., Donati, M., \& Hamilton, J. (2015). The development and testing of a new version of the Cognitive Reflection Test applying item response theory (IRT). Journal of Behavioral Decision Making. doi:10.1002/bdm.1883

Sinayev, A., \& Peters, E. (2015). Cognitive reflection vs. calculation in decision making. Frontiers in Psychology, 6, 532.

Stanovich, K. E., \& West, R. F. (2000). Individual differences in reasoning: Implications for the rationality debate? Behavioral and Brain Sciences, 22, 5, 645-726.

Stieger, S., Burger, C., Schiller, F. R., Schulze, E. K., \& Voracek, M. (2014). Measuring implicit gender-role orientation: The Gender Initial Preference Task. Journal of Personality Assessment, 96, 358-367.

Toplak, M. E., West, R. F., \& Stanovich, K. E. (2011). The Cognitive Reflection Test as a predictor of performance on heuristics-and-biases tasks. Memory and Cognition, 39, 12751289.

Toplak, M. E., West, R. F., \& Stanovich, K. E. (2014). Assessing miserly information processing: An expansion of the Cognitive Reflection Test. Thinking and Reasoning, 20, 147-168. doi:10.1080/13546783.2013.844729 
410 Weller, J. A., Dieckmann, N. F., Tusler, M., Mertz, C. K., Burns, W. J., \& Peters, E. (2013).

411 Development and testing of an abbreviated numeracy scale: A Rasch analysis approach.

412 Journal of Behavioral Decision Making, 26, 198-212.

413 Welsh, M., Burns, N., \& Delfabbro, P. (2013). The Cognitive Reflection Test: How much more

414 than numerical ability? In M. Knauff, N. Sebanz, M. Pauen, \& I. Wachsmuth (Eds.),

415 Proceedings of the 35th Annual Meeting of the Cognitive Science Society (pp. 1587-1592).

416 Cognitive Science Society.

417 Williams, R. L. (2013). Overview of the Flynn effect. Intelligence, 41, 753-764.

418 doi:10.1016/j.intell.2013.04.010

419 